\title{
Combination of dirty mass volume and APACHE II score predicts mortality in patients with colorectal perforation
}

Daichi Ishikawa, Yukako Takehara, Atsushi Takata, Kazuhito Takamura and Hirohiko Sato

Department of Surgery, Yoshinogawa Medical Center

Correspondence to: Daichi Ishikawa, MD, PhD, Department of Surgery, Yoshinogawa Medical Center, 120 Nishichiejima, Yoshinogawa City, Tokushima 776-8511, Japan. Tel: +81 883262222, Fax: +81 883262300, e-mail: daichi19850125@hotmail.co.jp

Article type: Original article

Keywords: Colorectal perforation, Dirty mass, APACHE II, Prognostic factor 


\section{Abstract}

Background: "Dirty mass" is a specific computed tomography (CT) finding that is seen frequently in colorectal perforation. The prognostic significance of this finding for mortality is unclear.

Methods: Fifty-eight consecutive patients with colorectal perforation who underwent emergency surgery were included in the study. Dirty mass identified on multidetector row CT (MDCT) was 3D-reconstructed and its volume was calculated using Ziostation software. Dirty mass volume and other clinical characteristics were compared between survivor $(n=45)$ and mortality groups $(n=13)$ to identify predictive factors for mortality. Mann-Whitney $U$ test and $X^{2}$ test were used in univariate analysis and logistic regression analysis was used in multivariate analysis.

Results: Dirty mass was identified in $36 / 58$ patients $(62.1 \%)$ and located next to perforated colorectum in all cases. Receiver-operating characteristic (ROC) curve analysis identified the highest peak at $96.3 \mathrm{~cm}^{3}$, with sensitivity of 0.643 and specificity of 0.864 . Univariate analysis revealed dirty mass volume, acute disseminated intravascular coagulation (DIC) score, acute physiology and chronic health evaluation II (APACHE II) score, and sequential organ failure assessment (SOFA) score as prognostic markers for mortality $(p<0.01)$. Multivariate analysis revealed dirty mass volume and APACHE II score as independent prognostic indicators for mortality. Mortality was stratified by dividing patients into four groups according to dirty mass volume and APACHE II score.

Conclusions: The combination of dirty mass volume and APACHE II score could stratify the postoperative mortality risk in patients with colorectal perforation. According to the risk stratification, surgeons might be able to decide the surgical procedures and intensity of postoperative management. 


\section{Introduction}

Colorectal perforation is a emergent status that causes severe sepsis, disseminated intravascular coagulation (DIC), and multiple organ failure (1). Despite recent advances in surgical techniques and intensive perioperative care, mortality rates remain high $(2,3)$. Thus, it is important to predict postoperative outcome by conducting preoperative examinations and evaluating the patient's condition. Predictive factors include such as older age, low preoperative blood pressure, arterial blood lactate concentration; as well as scoring systems such as acute physiology and chronic health evaluation II (APACHE II) score, sequential organ failure assessment (SOFA) score, and physiological and operative severity for the enumeration of mortality and morbidity (POSSUM) score (4-7).

Multidetector computed tomography (MDCT) is a rapid imaging modality that is commonly used in the setting of acute abdominal emergency, and is the most reliable modality for diagnosing colorectal perforation (8). Focal collection of extraluminal fecal matter is a specific finding of colorectal perforation that can be identified on MDCT. Saeki et al first reported extraluminal stool as "dirty mass" and described its characteristics (9). However, the significance of dirty mass on the severity of colorectal perforation and on patient prognosis has not been elucidated. The aims of the present study were to quantify dirty mass volume and investigate its association with mortality, together with other previously reported prognostic factors and scoring systems.

\section{Materials and Methods}

\section{Patients}


Fifty-eight consecutive patients with colorectal perforation who underwent emergency surgery at the Department of Surgery in Yoshinogawa Medical Center between 2012 and 2020 were enrolled in the study. Patients with perforation of the appendix or iatrogenic perforation were excluded from the study. Patients' clinical data, including medical history, physical examination and preoperative laboratory data, and details of treatments were retrospectively reviewed from the medical records. The patients' characteristics are listed in Table 1. At 30 days after emergency surgery, 45 patients $(77.6 \%)$ survived and $13(22.4 \%)$ had died. The patients were divided into a survivor group and a mortality group for further analysis. Patient characteristics and therapeutic factors were compared between the survivor and mortality groups (Tables 2 and 3 ). Body temperature was separated into $\leq 36^{\circ}$, $36^{\circ} \mathrm{C}-38^{\circ} \mathrm{C}$, and $>38^{\circ} \mathrm{C}$, and white blood cell counts into $\leq 4000 / \mu \mathrm{l}, 4000 / \mu \mathrm{l}-$ $12,000 / \mu \mathrm{l}$, and $>12,000 / \mu \mathrm{l}$ according to the criteria for systemic inflammatory response syndrome(10). Scoring systems such as the acute DIC score established by Japanese Association for Acute Medicine, and APACHE II, SOFA, and POSSUM scores were calculated preoperatively.

\section{Reconstruction and quantification of dirty mass}

All patients underwent MDCT prior to emergency surgery and the images were assessed for the presence of colorectal perforation and dirty mass by two radiologists. Dirty mass was defined as a localized area of low attenuation containing conglomerate air bubbles, as described previously(9). A 3D reconstruction of the dirty mass was obtained from the MDCT data using Ziostation software (Ziosoft, Tokyo, Japan). As the mass could not be traced automatically by the software, tracing was done manually at $1 \mathrm{~mm}$ intervals on axial images, followed 
by $3 \mathrm{D}$ reconstruction (Figure 1) and dirty mass volume $\left(\mathrm{cm}^{3}\right)$ was then calculated using the software.

\section{Treatment}

Following the confirmation of colorectal perforation intraoperatively, the surgical procedure was selected as follows. Resection of the diseased colonic segment with end colostomy Hartmann's procedure (HP) was performed for perforation sites in the left-sided colon or rectum, or if the patient's condition required a vasopressor agent during surgery. If the perforation site was right sided and the circulatory dynamics were stable, colectomy and primary anastomosis (PA) were performed. If the perforation site was unresectable due to cancer invasion or there was a relatively small perforation without severe inflammatory change around the perforation, stoma creation was performed. Open abdominal surgery was not carried out in any patient. Ventilation was continued after surgery until recovery from septic shock in the case of unstable circulatory condition, or if $>50 \%$ oxygen concentration was required to maintain saturation of $>90 \%$. Polymyxin Bimmobilized fiber direct hemoperfusion (PMX-DHP) was conducted when circulatory dynamics were unstable postoperatively even after the use of a vasopressor. Human recombinant thrombomodulin was administered if acute DIC score was $\geq 4$ points.

\section{Statistical analysis}

All analyses were conducted using the JMP 10 statistical software package (SAS Institute Inc., Tokyo, Japan). Receiver-operating characteristic (ROC) curve analysis was used to determine the cut-off point for dirty mass. Mann-Whitney Utest was used for comparison of continuous variables. The chi-squared test was 
used to analyze the relationship of clinical characteristics. Multivariate analysis was conducted by a logistic regression analysis using factors with $p$ value $<0.01$ in the univariate analysis. A $p$ value $<0.05$ was considered to indicate statistically significant difference.

\section{Results}

\section{Significance of dirty mass on prognosis}

Figure $1 \mathrm{~A}$ and $1 \mathrm{~B}$ show manual tracing and $3 \mathrm{D}$ reconstruction of a dirty mass for volume calculation. The positional relationship between the dirty mass and the colorectum was easily recognized on the 3D image. Dirty mass was identified in $36 / 58$ cases $(62.1 \%)$; in all 36 cases, dirty mass was associated with perforated colorectum. Dirty mass volume was significantly larger in the mortality group than in the survivor group (Figure 2A). The ROC curve showed the highest peak at volume of $96.3 \mathrm{~cm}^{3}$, with 0.643 sensitivity and 0.864 specificity (Figure 2B).

\section{Factors of colorectal perforation predictive of mortality}

Table 2 shows the comparison of patient background, physical examination and disease factors between the survivor and mortality groups. The mortality group had significantly higher age, American Society of Anesthesiologists-physical status (ASA-PS) score, and frequency of preoperatively coexisting comorbidity; and significantly lower mean arterial pressure (MAP) compared with the survivor group. Table 3 shows the comparison of therapeutic factors, examination data, and scores between the survivor and mortality groups. The mortality group had significantly lower frequency of ventilator use and thrombomodulin use; and significantly higher dirty mass volume, DIC score, APACHE II score, SOFA score and POSSUM score compared with the survivor group. 
Univariate analysis revealed significant differences with $p<0.01$ for dirty mass volume, DIC score, APACHE II score and SOFA score, and these four potential prognostic factors underwent further multivariate analysis. Logistic regression analysis revealed dirty mass volume and APACHE II score as independent prognostic factors, with significant difference, as shown in Table 4.

\section{Stratification of mortality risk}

Using the results of multivariate analysis, we divided the patients into four groups according to dirty mass volume and APACHE II score (Figure 3): high volume / low score (group A), high volume / high score (group B), low volume / low score (group C) and low volume / high score (group D). Based on the ROC curve shown in Figure 2B, a cut-off value of 100 was used for dirty mass volume, and the median APACHE II value of 11 was selected to assign high/low score. Mortality was $3 / 7(42.8 \%)$ in group $A, 6 / 9(66.6 \%)$ in group $B, 0 / 22(0 \%)$ in group $C$, and 4/20 $(20.0 \%)$ in group D. These results indicate that combined dirty mass volume and APACHE II score can be used to stratify the mortality risk of patients with colorectal perforation.

\section{Discussion}

Dirty mass was initially reported by Saeki et al as the CT finding of a focal collection of extraluminal fecal matter(9). In their study, CT depicted this appearance in $15 / 29$ cases $(51.7 \%)$, size was variable (range, $1-6 \mathrm{~cm}$ ), and dirty mass was located very close to the site of perforation. The present study is the first to undertake $3 \mathrm{D}$ reconstruction and to quantify dirty mass volume. 3D imaging enables surgeons to easily recognize the positional relationship between dirty mass and perforated colon and to plan the surgical procedure accurately. We identified dirty mass in $36 / 58$ of the present cases $(62.1 \%)$, all of which were located next to 
the perforated colorectum, consistent with the data published by Saeki et al. Furthermore, univariate and multivariate analysis revealed that dirty mass is a potential prognostic predictor.

In addition to dirty mass volume, the present univariate analysis identified the following as prognostic factors or scoring systems: older age, higher ASA-PS score, preoperative coexisting comorbidity, lower MAP, ventilator use, thrombomodulin use, higher DIC score, higher APACHE II score, higher SOFA score, and higher POSSUM score. Several predictive factors and scoring systems have been proposed for postoperative outcome in patients with colorectal perforation. Yamamoto et al identified older age and low preoperative blood pressure as routinely available prognostic markers in colorectal perforation(4). Harries et al reported that preoperative ASA-PS and hemoglobin were significant independent predictors of mortality in perforated sigmoid diverticular disease(11), although there was no difference in hemoglobin levels between the present survivor and mortality groups. Scoring systems provide objective and systematic assessment of the severity of colorectal perforation, and various reports have described the utility of scoring systems for predicting the mortality risk of colorectal perforation. Nakamura et al reported acute DIC score as the strongest predictor of mortality by multivariate analysis(12); and reported SOFA and POSSUM scores as significant markers in univariate analysis, but not APACHE II ( $p=0.053)$. SOFA and POSSUM scores have been reported as useful prognostic markers for colorectal perforation in other studies $(13,14)$. APACHE II is a disease-independent score for evaluation of highly dependent patients in the intensive care unit. Horiuchi et al reported that APACHE II score was most strongly associated with poor prognosis, and reported mortality in $>80 \%$ of patients with APACHE II score $>15$ and all patients with APACHE II score $>20(6)$. In the present study, patients were divided according to APACHE II score at 
a median value of 11 . When the patients were divided at different cut-off values, the mortality rate was $53 \%$ in patients with APACHE II score $>15$ and $83 \%$ in patients with APACHE II score >20 (Figure 3), compatible with the findings of Horiuchi et al. Regarding ventilator and thrombomodulin use, these therapeutic choices were made in patients with severe conditions such as septic shock and DIC, which could have affected outcome in the mortality group.

Colorectal perforation followed by severe peritonitis requires emergency surgery such as HP and $\mathrm{PA}(15,16)$. There is no consensus regarding the most suitable surgical procedure and the indication for the procedure is commonly decided by the individual surgeon depending on the situation. In their propensityscore matched model, Tsuchiya et al reported a higher rate of mortality for PA than for HP, and recommended that HP should be selected for patients with shock, immunosuppressive conditions, or advanced age(17). Recently, less invasive approaches such as laparoscopic lavage have been reported as an effective alternative, although this procedure has a high rate of total reoperations and subsequent percutaneous drainage(18). In the present study, mortality risk was stratified according to the combination of dirty mass volume and APACHE II score as an indicator of the severity of panperitonitis and the patient's general condition. This stratification might enable surgeons to predict mortality risk and perform PA or even laparoscopic lavage for patients with low risk. However, further prospective study is required to evaluate the most appropriate therapeutic strategy.

In conclusion, the findings of our study revealed the dirty mass volume as a potential prognostic indicator and that the combination of dirty mass volume and APACHE II score could stratify the postoperative mortality risk of patients with colorectal perforation. According to the risk stratification, surgeons might be able to 
decide the surgical procedures and could perform intensive postoperative management.

\section{List of abbreviations}

MDCT: multi-detector row computed tomography; ROC: receiver-operating characteristic; DIC: disseminated intravascular coagulation; APACHE II: acute physiology and chronic health evaluation II; SOFA: sequential organ failure assessment; POSSUM: physiological and operative severity score for the enumeration of mortality and morbidity; PMX-DHP: polymyxin B-immobilized fiber direct hemoperfusion; ASA-PS: American Society of Anesthesiologists-physical status; MAP: mean arterial pressure; HP: Hartmann's procedure; PA: primary anastomosis

\section{Declarations}

Ethics approval and consent to participate: The study protocol was approved by the Ethics Committee of Yoshinogawa Medical Center (No. 20201201).

Consent for publication: Informed consent was obtained from all participating patients. In the case of deceased patients, consent was obtained from consanguine family or a legal guardian.

Availability of data and materials: The dataset supporting the conclusions of this article is included within the article and additional file.

Competing interests: The authors declare that they have no competing interests.

Funding: None 
Authors' contributions: Ishikawa D designed the study, acquired and analyzed the data and wrote the initial draft of the article. Takehara $\mathrm{Y}$ and Takata $\mathrm{A}$ contributed to analysis and interpretation of data. Takamura $\mathrm{K}$ assisted in preparation of the article. Sato H supervised the project. All authors have critically reviewed the manuscript and approved the final version.

Acknowledgements: The authors would like extend their thanks to Tanagami Tomoaki, Shimazu Hideki, and Matsushita Tomoki for diagnostic support with computed tomography and to Yasuhiro Funakoshi for help with use of the Ziostation software. 


\section{References}

1. Wood CD. Acute perforations of the colon. Diseases of the colon and rectum. 1977;20:126-9.

2. Bielecki K, Kamiński P, Klukowski M. Large bowel perforation: morbidity and mortality. Techniques in coloproctology. 2002;6:177-82.

3. Tan KK, Hong CC, Zhang J, Liu JZ, Sim R. Predictors of outcome following surgery in colonic perforation: an institution's experience over 6 years. Journal of gastrointestinal surgery : official journal of the Society for Surgery of the Alimentary Tract. 2011;15:277-84. 4. Yamamoto T, Kita R, Masui H, Kinoshita H, Sakamoto Y, Okada K, et al. Prediction of mortality in patients with colorectal perforation based on routinely available parameters: a retrospective study. World journal of emergency surgery : WJES. 2015;10:24.

5. Shimazaki J, Motohashi G, Nishida K, Ubukata H, Tabuchi T. Postoperative arterial blood lactate level as a mortality marker in patients with colorectal perforation. Int J Colorectal Dis. 2014;29:51-5.

6. Horiuchi A, Watanabe Y, Doi T, Sato K, Yukumi S, Yoshida M, et al. Evaluation of prognostic factors and scoring system in colonic perforation. World journal of gastroenterology: WJG. 2007;13:3228-31.

7. Sumi T, Katsumata K, Katayanagi S, Nakamura Y, Nomura T, Takano K, et al. Examination of prognostic factors in patients undergoing surgery for colorectal perforation: a case controlled study. International journal of surgery (London, England). 2014;12:566-71. 8. Kim SH, Shin SS, Jeong YY, Heo SH, Kim JW, Kang HK. Gastrointestinal tract perforation: MDCT findings according to the perforation sites. Korean journal of radiology. 2009;10:63-70.

9. Saeki M, Hoshikawa Y, Miyazaki O, Nakayama F, Okamoto E, Ishikawa T. Computed tomographic analysis of colonic perforation: "Dirty mass," a new computed tomographic finding. Emergency Radiology. 1998;5:140-5.

10. Bone RC, Balk RA, Cerra FB, Dellinger RP, Fein AM, Knaus WA, et al. Definitions for sepsis and organ failure and guidelines for the use of innovative therapies in sepsis. The ACCP/SCCM Consensus Conference Committee. American College of Chest Physicians/Society of Critical Care Medicine. Chest. 1992;101:1644-55.

11. Harries RL, Twine CP, Kugathasan G, Young H, Jones E, Gomez KF. Prognostic factors for survival following emergency Hartmann's procedure. Postgraduate medical journal. 2012;88:205-9.

12. Nakamura F, Yui R, Muratsu A, Sakuramoto K, Muroya T, Ikegawa H, et al. Study of the prognostic factor of the colon perforation case with the pan-peritonitis that needed emergency surgery: a single-center observational study. Acute medicine \& surgery. 2019;6:379-84.

13. Ochiai T, Hiranuma S, Takiguchi N, Ito K, Kawaguchi A, Iwai T, et al. SOFA score predicts postoperative outcome of patients with colorectal perforation. Hepatogastroenterology. 2004;51:1007-10.

14. Ishizuka M, Nagata H, Takagi K, Horie T, Kubota K. POSSUM is an optimal system for predicting mortality due to colorectal perforation. Hepato-gastroenterology. 2008;55:430-3.

15. Trenti L, Biondo S, Golda T, Monica M, Kreisler E, Fraccalvieri D, et al. Generalized peritonitis due to perforated diverticulitis: Hartmann's procedure or primary anastomosis? Int J Colorectal Dis. 2011;26:377-84.

16. Moore FA, Catena F, Moore EE, Leppaniemi A, Peitzmann AB. Position paper: management of perforated sigmoid diverticulitis. World journal of emergency surgery : WJES. 2013;8:55.

17. Tsuchiya A, Yasunaga H, Tsutsumi Y, Matsui H, Fushimi K. Mortality and Morbidity After Hartmann's Procedure Versus Primary Anastomosis Without a Diverting Stoma for 
Colorectal Perforation: A Nationwide Observational Study. World journal of surgery. 2018;42:866-75.

18. Galbraith N, Carter JV, Netz U, Yang D, Fry DE, McCafferty M, et al. Laparoscopic Lavage in the Management of Perforated Diverticulitis: a Contemporary Meta-analysis. Journal of gastrointestinal surgery : official journal of the Society for Surgery of the Alimentary Tract. 2017;21:1491-9. 


\section{Figure legends}

Figure 1. 3D reconstruction and quantification of dirty mass with Ziostation software.

A: The position of a dirty mass is indicated by the yellow dashed line that was handtraced on an axial MDCT image. These outlines were used to construct a 3D image.

B: Representative 3D image of a dirty mass constructed using Ziostation. The dirty mass (green) is located next to the sigmoid colon (pink). The volume of the dirty mass was calculated as $419.2 \mathrm{~cm}^{3}$.

Figure 2. Comparison of dirty mass volume between the survivor and mortality groups.

A: Dirty mass volume was significantly larger in the mortality group than the survivor group.

B: ROC curve shows the highest peak at dirty mass volume of $96.3 \mathrm{~cm}^{3}$, with 0.643 sensitivity and 0.864 specificity.

Figure 3. Stratification of mortality risk according to dirty mass volume and APACHE II score.

Patients with colorectal perforation were divided into four groups ( $A$ to $D$ ) according to various combinations of dirty mass volume and APACHE II score. The mortality rates for groups $A, B, C$ and $D$ were $42.8 \%, 66.6 \%, 0 \%$, and $20.0 \%$, respectively. 
Table 1. Patient characteristics

\begin{tabular}{lr}
\hline Factor & $(\mathrm{n}=58)$ \\
\hline Age (years) & $80(36-94)$ \\
Male & $29(50)$ \\
BMI & $21.7 \pm 3.8$ \\
ASA-PS & \\
I & $6(10.3)$ \\
II & $28(48.3)$ \\
III & $22(37.9)$ \\
IV & $2(3.4)$ \\
Survived / Mortality & $13(22.4)$ \\
Comorbidity & $38(65.5)$ \\
Steroid & $6(10.3)$ \\
Cause & \\
Diverticulitis & $24(41.4)$ \\
Cancer & $18(31.0)$ \\
Ischemia & $6(10.3)$ \\
Idiopathic & $5(8.6)$ \\
Other & $5(8.6)$ \\
Perforation site & \\
Ascending colon & $9(15.5)$ \\
Transverse colon & $7(12.1)$ \\
Descending colon & $4(6.9)$ \\
Sigmoid colon & $28(48.3)$ \\
Rectum & $10(17.2)$ \\
Length of operation (min) & $114(54-182)$ \\
Blood loss (ml) & $59(10-1500)$ \\
\hline
\end{tabular}

Data are expressed as the median (range), mean \pm standard deviation, or $\mathrm{n}(\%)$. BMI: body mass index; ASA-PS: American Society of Anesthesiologists-Physical Status 
Table 2. Comparison of patient background, physical examination, and disease factors between the survivor and mortality groups

\begin{tabular}{|c|c|c|c|}
\hline & Survivors & Mortality & $p$ value \\
\hline & $(n=45)$ & $(n=13)$ & \\
\hline Age (years) & $79(36-94)$ & $82(68-90)$ & $0.042^{*}$ \\
\hline Male & 23 & 6 & 1.000 \\
\hline $\mathrm{BMI}\left(\mathrm{kg} / \mathrm{m}^{2}\right)$ & $21.9 \pm 4.0$ & $21.1 \pm 2.4$ & 0.632 \\
\hline \multicolumn{4}{|l|}{ ASA-PS } \\
\hline I & 6 & 0 & \\
\hline ॥ & 25 & 3 & \\
\hline III & 13 & 9 & \\
\hline IV & 1 & 1 & $0.028 *$ \\
\hline Comorbidity & 26 & 12 & $0.048 *$ \\
\hline Steroid use & 4 & 2 & 0.873 \\
\hline Heart rate $(/ \mathrm{min})$ & $94(56-134)$ & $100(54-143)$ & 0.081 \\
\hline MAP $(\mathrm{mmHg})$ & $88 \pm 17$ & $75 \pm 20$ & $0.023 *$ \\
\hline Body temperature $>38.0^{\circ} \mathrm{C}$ & 21 & 5 & 0.836 \\
\hline \multicolumn{4}{|l|}{ or $<36.0^{\circ} \mathrm{C}$} \\
\hline Time from onset to surgery & 18 & 2 & 0.189 \\
\hline \multicolumn{4}{|l|}{ Cause } \\
\hline Diverticulitis & 3 & 21 & \\
\hline Cancer & 6 & 12 & \\
\hline Idiopathic & 0 & 5 & \\
\hline Ischemia & 2 & 4 & \\
\hline Other & 2 & 3 & 0.255 \\
\hline \multicolumn{4}{|l|}{ Perforation site } \\
\hline Ascending colon & 6 & 3 & \\
\hline Transverse colon & 6 & 1 & \\
\hline Descending colon & 3 & 1 & \\
\hline Sigmoid colon & 22 & 6 & \\
\hline Rectum & 8 & 2 & 0.922 \\
\hline
\end{tabular}

Data are expressed as the median (range), mean \pm standard deviation.

$* p<0.05$ 
BMI: body mass index; ASA-PS: American Society of Anesthesiologists-Physical Status; MAP: mean arterial pressure Comorbidities include diabetes mellitus, heart, lung, or renal disease. 
Table 3. Comparison of therapeutic factors, examination data, and score values between the survivor and mortality groups

\begin{tabular}{llll}
\hline & $\begin{array}{l}\text { Survivors } \\
(\mathrm{n}=45)\end{array}$ & $\begin{array}{l}\text { Mortality } \\
(\mathrm{n}=13)\end{array}$ & $p$ value \\
\hline Procedure & & & \\
$\quad$ Hartmann's procedure & 30 & 12 & \\
$\quad$ Colectomy and PA & 11 & 1 & \\
$\quad$ Stoma creation & 4 & 0 & 0.179 \\
Length of operation & $118(54-182)$ & $106(72-152)$ & 0.837 \\
Blood loss & $60(10-1500)$ & $59(10-324)$ & 0.747 \\
Transfusion & 20 & 5 & 0.948 \\
Ventilator use & 16 & 10 & $0.020^{*}$ \\
PMX-DHP use & 15 & 7 & 0.309 \\
IVIG use & 12 & 7 & 0.133 \\
Thrombomodulin use & 18 & 10 & $0.042^{*}$ \\
Hemoglobin $(\mathrm{g} / \mathrm{dl})$ & $12.1 \pm 2.5$ & $12.5 \pm 2.8$ & 0.608 \\
WBC $>12000$ or $<4000(/ \mu \mathrm{l})$ & 23 & 10 & 0.181 \\
Platelet $\left(\times 10^{3} / \mu \mathrm{l}\right)$ & $249 \pm 120$ & $219 \pm 100$ & 0.439 \\
CRP $(\mathrm{mg} / \mathrm{dl})$ & $12.3 \pm 11.8$ & $6.4 \pm 8.3$ & 0.156 \\
Albumin $(\mathrm{mg} / \mathrm{dl})$ & $3.3 \pm 0.7$ & $3.0 \pm 0.6$ & 0.167 \\
Creatinine $(\mathrm{mg} / \mathrm{dl})$ & $1.5 \pm 1.6$ & $2.4 \pm 2.2$ & 0.060 \\
Dirty mass volume $\left(\mathrm{cm}{ }^{3}\right)$ & $54 \pm 100$ & $234 \pm 211$ & $0.004^{* *}$ \\
DIC score & $1(0-6)$ & $3(0-8)$ & $0.007^{* *}$ \\
APACHE II score & $11(3-22)$ & $18(8-30)$ & $0.001^{* *}$ \\
SOFA score & $2(0-6)$ & $5(0-12)$ & $0.002^{* *}$ \\
POSSUM score & $44(29-64)$ & $53(34-74)$ & $0.030^{*}$ \\
\hline
\end{tabular}

Data are expressed as the median (range), mean \pm standard deviation. $* p<0.05, * * p<0.01$

PA: primary anastomosis; PMX-DHP: polymyxin B-immobilized fiber direct hemoperfusion; IVIG: intravenous immunoglobulin; WBC: white blood cells; CRP: C-reactive protein; DIC; disseminated intravascular coagulation; APACHE II: acute physiology and chronic health evaluation; SOFA: sequential organ failure 
assessment; POSSUM; physiological and operative severity score for the enumeration of mortality and morbidity. 
Table 4. Multivariate analysis of factors prognostic for colorectal perforation

\begin{tabular}{lll}
\hline & Odds ratio & $p$ value \\
\hline Dirty mass volume & $1.010(1.000-1.020)$ & $0.006^{* *}$ \\
DIC score & $1.310(0.678-2.520)$ & 0.424 \\
APACHE II score & $1.400(1.070-1.850)$ & $0.015^{*}$ \\
SOFA score & $1.170(0.699-1.950)$ & 0.554
\end{tabular}

Values in parentheses are the $95 \%$ confidence interval.

$* p<0.05, * * p<0.01$

DIC; disseminated intravascular coagulation; APACHE II: acute physiology and chronic health evaluation; SOFA: sequential organ failure assessment; POSSUM; physiological and operative severity score for the enumeration of mortality and morbidity. 
Figure 1

A

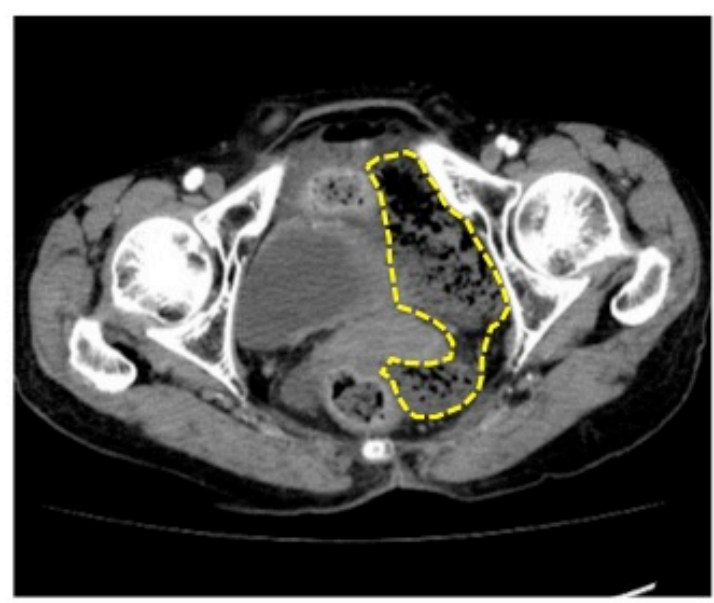

B

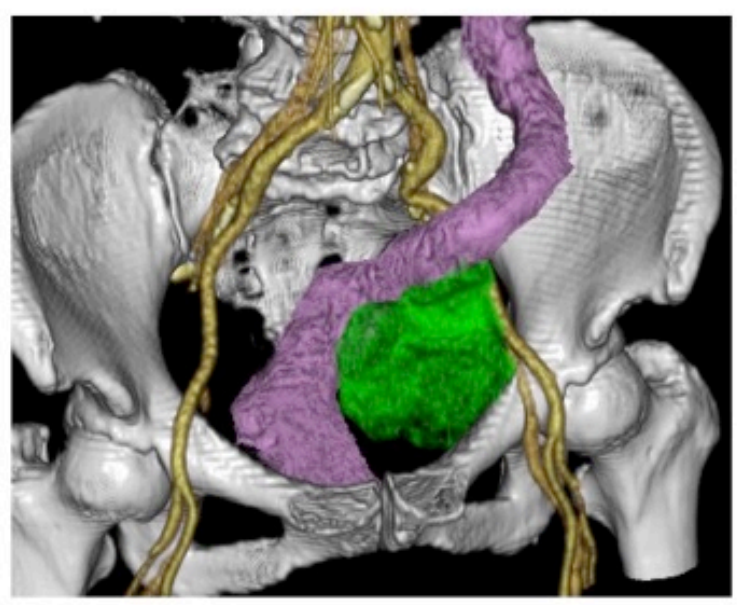


Figure 2

A

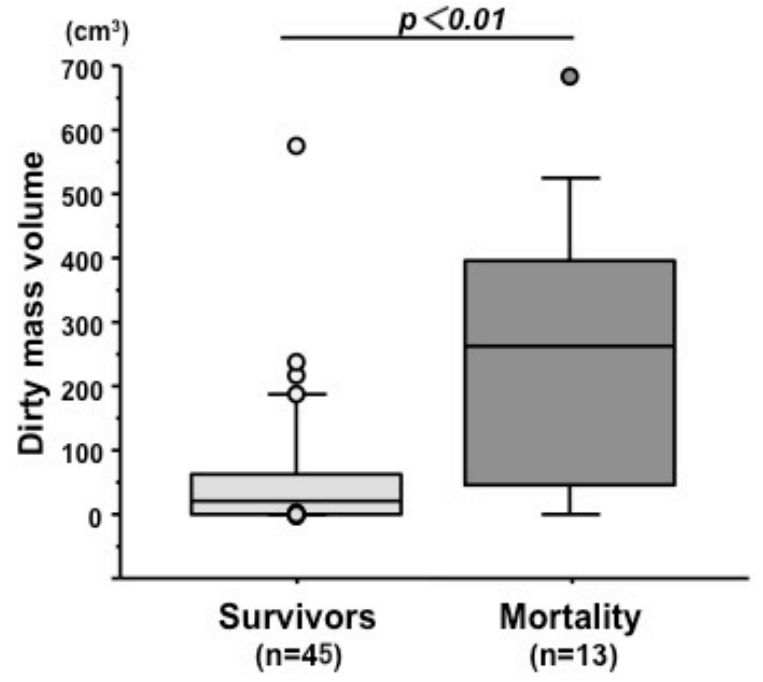

B

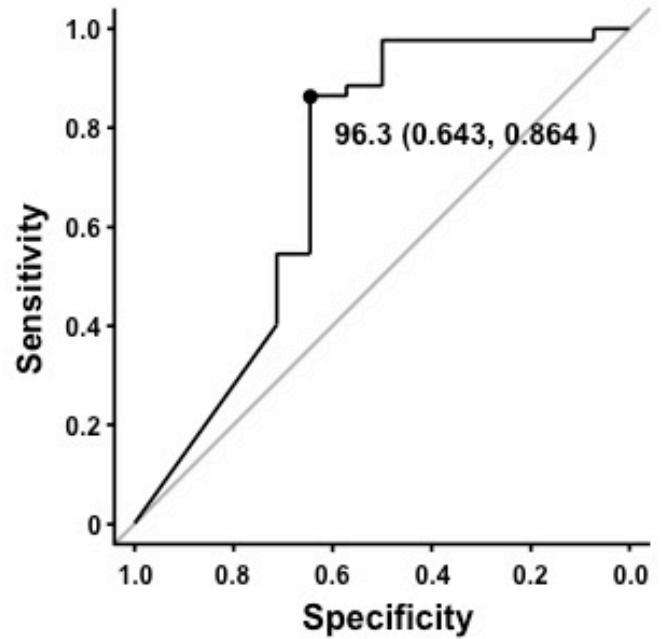

Figure 3

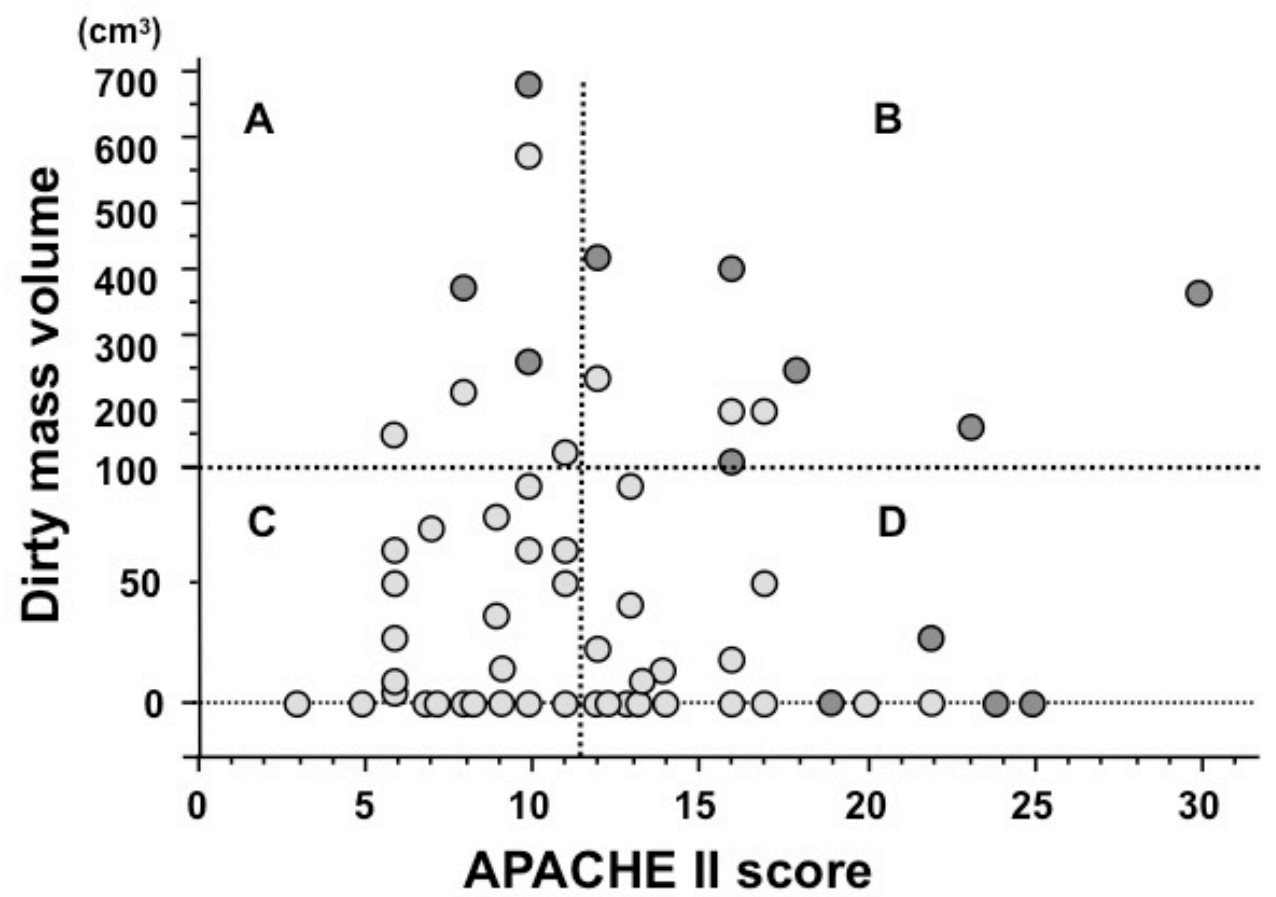

